\title{
Engineering Two-dimensional Materials Surface Chemistry
}

\author{
Chih-Jen Shih*
}

\begin{abstract}
This account reviews our recent research activities and achievements in the field of two-dimensional (2D) materials surface chemistry. 2D materials are atomically thin, so that carriers are less-restricted to move in the in-plane direction, whereas the out-of-plain motion is quantum-confined. Semiconductor quantum wells and graphene are two well-known examples. Applications of 2D materials in optoelectronics, surface modification, and complex materials must overcome engineering challenges associated with understanding and engineering surface chemistry of 2D materials, which essentially bridge multiscale physical phenomena. In my research group, we understand and engineer broad aspects of chemistry and physics at nanomaterials surfaces for advancing nanomaterials-based technologies. The three main topics covered in this account are as follows: i) colloidal synthesis of stacking-controlled 2D materials, ii) wetting properties of 2D materials, and iii) engineering electronic transport at 2D materials-semiconductor interfaces.
\end{abstract}

Keywords: 2D materials · Graphene $\cdot$ Interface engineering

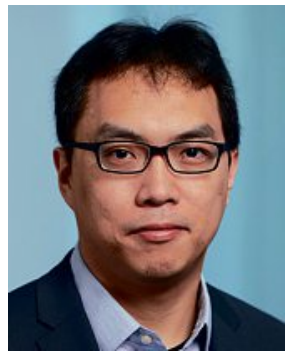

Chih-Jen Shih has been a tenure-track assistant professor of chemical engineering at the Department of Chemistry and Applied Biosciences, ETH Zurich since May, 2015. He received bachelor and master degrees from National Taiwan University (Taiwan, 1998-2004), followed by serving as a research engineer in a semiconductor company to fulfill his military obligation. Afterwards, he moved to the US and obtained his $\mathrm{PhD}$ degree from Massachusetts Institute of Technology (USA, 2009-2014). Prior to joining the faculty at ETH, he served as a postdoctoral researcher at Stanford University. He started the Laboratory for Interface and Surface Engineering of Nanomaterials (LISE) in the Institute for Chemical and Bioengineering (ICB) in 2015. The LISE is focused on the following directions: mesoscale modeling of nanomaterials, physics and chemistry at nanomaterials interfaces, nanomaterials processing, and nanomaterials-based devices.

${ }^{\star}$ Correspondence: Prof. Dr. C.-J. Shih Institute for Chemical and Bioengineering ETH Zurich

$\mathrm{CH}-8093$ Zurich

E-mail: chih-jen.shih@chem.ethz.ch

\section{Introduction}

In the past decade, two-dimensional (2D) materials have generated considerable research effort due to their unique physical properties, resulting from the confined charge and heat transport in the $2 \mathrm{D}$ structures. ${ }^{[1]}$ These materials are atomically thin, so that carriers are lessrestricted to move in the in-plane direction, whereas the out-of-plain motion is quantum-confined. ${ }^{[2]}$ The first 2D material, monolayer graphene, was first isolated by Geim and Novosolov using a thin transparent adhesive tape. ${ }^{[3]}$ The group further demonstrated the electronic properties of the one-atom-thick film by fabricating field-effect transistor (FET) devices. [3] Experimental findings not only corroborate that the monolayer form of $2 \mathrm{D}$ materials can stably exist at room temperature, but, more importantly, that the electronic properties of these materials may deviate significantly from those of the bulk form by reducing the layer number to a high degree (typically, <10 layers).[4]

Fig. 1 summarizes different types of $2 \mathrm{D}$ materials. ${ }^{[2]}$ The simplest molecular forms are monolayer graphene and hexagonal boron nitride (h-BN), which are comprised of $s p^{2}$-bonded atoms. ${ }^{[4-7]}$ Vermiculite, which

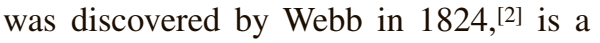
mineral similar to mica, as shown in Fig. 1D-F. Transition metal dichalcogenides (TMDs) (such as $\mathrm{MoS}_{2}$, see Fig. 1G-I ${ }^{[8]}$ and metal halides (such as $\left.\mathrm{PbI}_{2}\right)^{[9]}$ consist of a plane of metal atoms lying between two planes of halide/chalcogen atoms. Layered metal oxides, including $\mathrm{MnO}_{2}$, $\mathrm{MoO}_{3}$, and $\mathrm{LaNb}_{2} \mathrm{O}_{7}$ (see Fig. 1J-L), ${ }^{[10]}$ on the other hand, possess relatively complex molecular structures with a large degree of variety. Finally, layered silicates are also substances with many different types. A well-known example is the micas. ${ }^{[11]}$ Typically, the exfoliated oxides, $\mathrm{LDH}$, and silicates monolayers are charged and often attached by the counter ions. ${ }^{[10]}$ Other wellstudied systems include the III-VI compounds, such as InSe and GaS, ${ }^{12}$ the V-VI compounds, such as $\mathrm{Bi}_{2} \mathrm{Te}_{3}$ and $\mathrm{Sb}_{2} \mathrm{Se}_{3},{ }^{[13]}$ the metal trichalcogenides, and the metal trihalides.

As reflected in Fig. 1, the diversity of 2D materials appears to be one advantage. With the bulk forms themselves, different molecular combinations already result in a considerable degree of variation in physical properties. For example, the TMD ${ }^{[8]}$ family possess more than 40 different members considering different combinations of chalcogen ( $\mathrm{S}, \mathrm{Se}$, or Te) and transition metal. Furthermore, the coordination and oxidation state of the metal atoms also determine the resulting TMDs to be superconducting, metallic, semi-metallic, or semiconducting. ${ }^{[8]}$ Recent findings in thin-layer graphene have further stimulated considerable research in understanding and engineering the layer-dependent properties of TMDs. Next, I will briefly discuss three different projects in the field of 2D materials surface chemistry being carried out in my group.

\section{Colloidal Synthesis of Stacking- controlled 2D Materials}

Recent advances in 2D materials have suggested that not only the monolayer form is of interest, but also the other counter- 


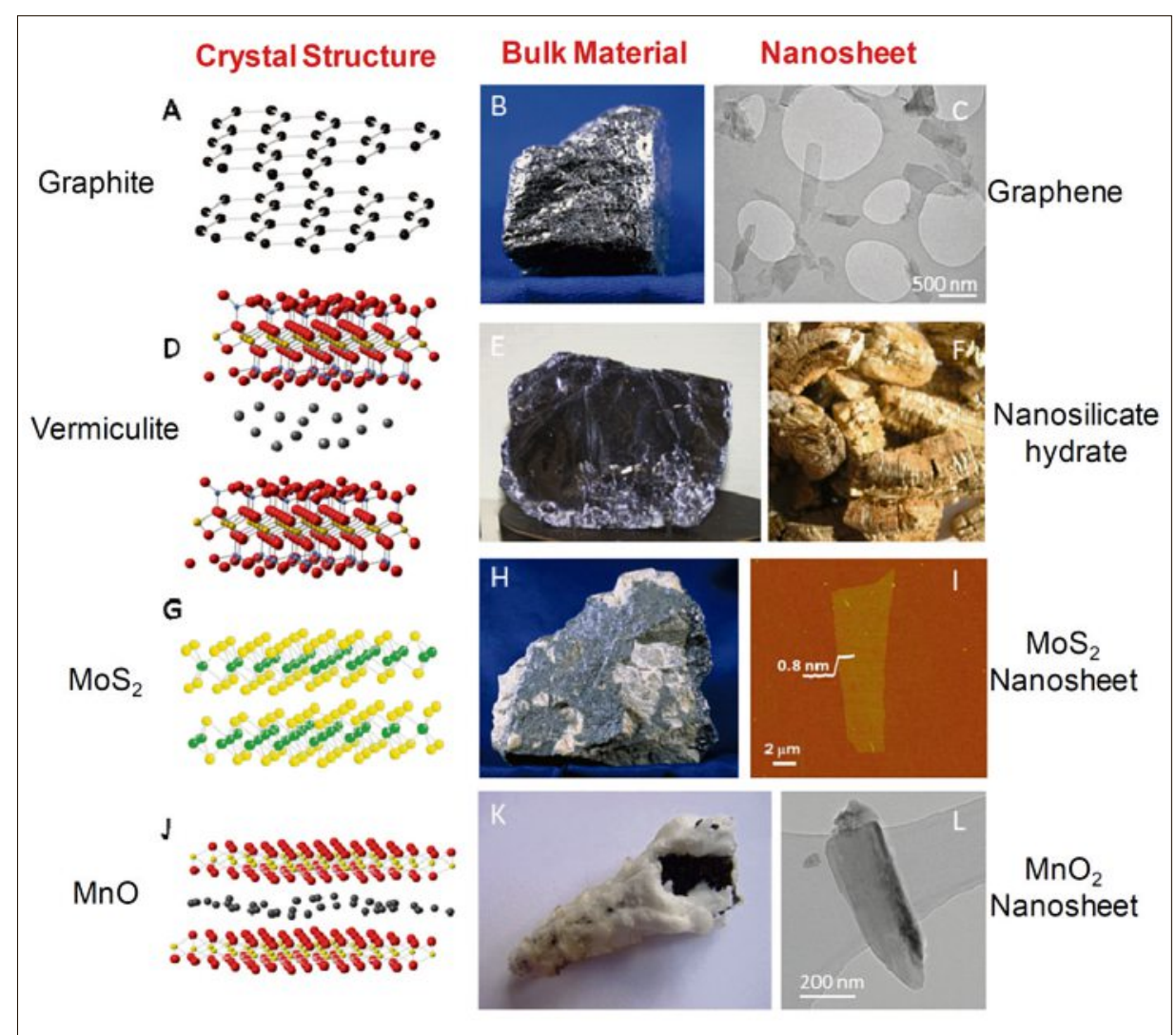

Fig. 1. Crystal structures, bulk forms, and exfoliated 2D sheets for four families of 2D materials. (A) Graphite consists of AB-stacked $s p^{2}$-hybridized carbon atoms (black beads), (B) is a mineral formed naturally, and (C) is the exfoliated monolayer graphene. (D) Vermiculite is a layered silicate hydrate that $(E)$ shows its mineral formed naturally and $(F)$ is the mineral after heating, allowing easy exfoliation. (Color code: silicon: blue; oxygen: red; Al/Mg/Fe: yellow; the interlayer counterions: black). (G) $\mathrm{MoS}_{2}$ is a layered Mo atoms sandwiched by two layers of $\mathrm{S}$ atoms (chalcogen: yellow, and transition metals: green) that $(\mathrm{H})$ is its mineral formed naturally, and (I) the exfoliated monolayer $\mathrm{MoS}_{2}$ sheets. $(\mathrm{J})$ Layered manganese dioxide (manganese: yellow; oxygen: red; and interlayer counterions: black) with its natural form $(\mathrm{K})$ and $(\mathrm{L})$ the exfoliated form of $\mathrm{MnO}_{2}$ nanosheets. ${ }^{[2]}$ Reprinted with permission from ref. [2]. Copyright 2013 Science Publishing Group.

parts with different stacking numbers can exhibit distinct physical properties. For example, when two graphene layers are placed together and form bilayer graphene, the energy barrier associated with the quantum mechanical hopping between the two layers of graphene is about $300 \mathrm{meV}$, resulting from the high-energy electronic sub-bands. ${ }^{[14]}$ The high-energy states are not involved in carrier transport unless a very high degree of doping is achieved. [14] Similar to monolayer graphene, the covalently bonded atoms result in a symmetric pair of low-energy bands, which cross at the Dirac point, but have parabolic $E-k$ dispersion, and the symmetry in hole and electron transport is conserved. One can open a bandgap by breaking the symmetry. In the bilayer graphene system, by simply applying a vertical electric field, which is perpendicular to the graphene plane, one can break the symmetry and open a band gap, $E$, depending on the magnitude of the electric field. ${ }^{[15]}$ In this respect, bilayer graphene is one of few material systems in which the bandgap can be controlled by the magnitude of the electric field normal to great potential for next-generation optoelectronics. Nature Publishing Group.
Nevertheless, new synthesis methods are required for effective control over the stacking number in graphene on a large scale. For different material systems, we apply: i) the top-down approach that engineers exfoliation of the bulk counterpart via intercalation chemistry, and ii) the bottom-up approach that engineers molecular self-assembly from the reaction precursors via colloidal chemistry, as shown in Fig. 2. For example, regarding approach i), here we discuss the approach we developed for the graphene system. ${ }^{[16]}$ Graphite exfoliation in a liquid phase can be easily scalable as one of many 'conducting inks', amenable to printing technologies, and also accesses to precise surface functionalization. ${ }^{[17,18]}$ If layering can be manipulated in a top-down manner, this would provide a facile route for the mass-production of layer-controlled graphene on a variety of substrates for device applications.

We proposed a new concept that, when combined with the well-developed chemistry of graphite intercalation compounds (GICs), ${ }^{[19,20]}$ a high degree of layer control becomes possible, which corresponds to the blue curve in Fig. 2A. Specifically, we demonstrate that intercalation of ionic, non-covalent intercalants in graphite yields the Stage-2 and Stage-3 GICs (Fig. 2B). The staging mechanism corresponds to the long-range lattice strain effect.[19] When the exposed graphite surfaces interact with the intercalant molecules, the molecules diffuse into the bulk host material from the layers close to the exposed ends, and sequentially into the deep layers that are increasingly distant from the exposed ends of the GIC surfaces. The elastic force results in a decrease in strain energy by clustering and aligning neighboring intercalant molecules. ${ }^{[19]}$ We used the synthesized Stage- 2 and Stage- 3 GICs as precursors of expanded graphite (EG),

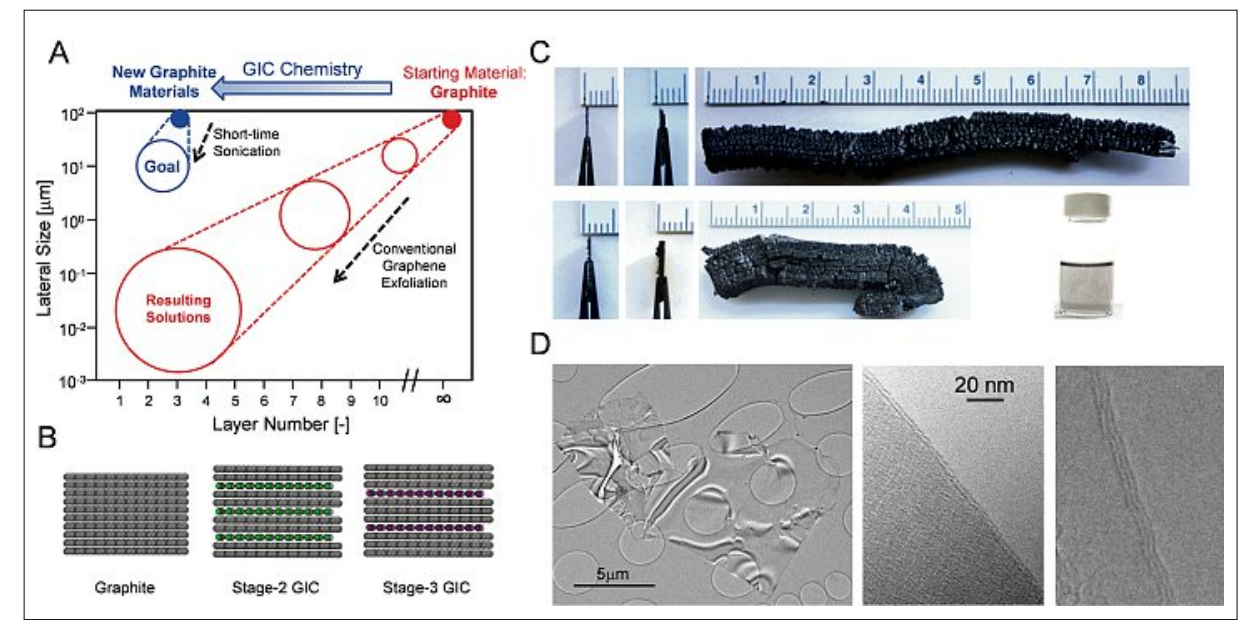

Fig. 2. Colloidal synthesis of layer-controlled graphene dispersions. (A) Schematic illustration of the concept. (B) Molecular models of graphite, Stage-2 GIC, and Stage-3 GIC. (Color code: black: carbon; green: chlorine; purple: iodine). Reprinted with permission from ref. [16]. Copyright 2011 
by placing the GICs in a high-temperature furnace. The non-covalently bonded intercalant molecules between graphene layers were vaporized rapidly and removed during the heating procedure. The degree of expansion exceeded several hundred times depending on the stage of the GIC (Fig. 2C).

We propose that the thermal expansion process isolates the bilayer and trilayer crystal domains that are crosslinked by grain boundaries and stacking faults formed during the intercalation process. Small sub-domains are expected to collapse and restack to form thicker and larger domains. Compared to the bulk graphite, where the graphene planes are stacked tightly by van der Waals (vdW) forces, the systematic, crosslinked defects that adhere bi-layer and tri-layer crystallites in the EGs are much easier to break in the following liquid exfoliation process. The resulting graphene dispersions would link to the GIC Stage number, and therefore creates a viable route towards bi- and trilayer graphene solutions. The voids inside the EG are expected to fill with Ar gas during the expansion process. Subsequently, a small amount of EGs were added in surfactant aqueous solution, followed by homogenization, sonication, and centrifugation. The gray and clear solution on the top part of the centrifuge tubes was finally decanted, which is our layer-controlled graphene solution, derived from the Stage- 2 or Stage- 3 GICs. Graphene microflakes are dispersed in the solutions, as confirmed by transmission electron microscopy (TEM) images (Fig. 2D).

\section{Wetting Properties of 2D Materials}

The production of large-area monolayer $2 \mathrm{D}$ material ${ }^{[21]}$ with chemical vapor deposition (CVD) has enabled large-area processing and applications. To integrate these materials into existing technologies, engineering interface properties is essential in processing of these materials. A trivial but unaddressed question is: "Can molecules residing on one side of monolayer 2D material experience electrostatic or van der Waals (vdW) interactions exerted by substances residing on the other side?" For example, in spite of the fact that monolayer $2 \mathrm{D}$ graphene only absorbs $2 \%$ of visible light, ${ }^{[22]}$ there is a lack of fundamental understanding about the degree of 'transparency' for 2D material to transmit chemical, $\mathrm{vdW}$, and electrostatic forces and interactions. Understanding wetting on graphene has been considered as the basis to quantify the effect of the one-atom-thick film, because the intermolecular interactions at the interface directly determine the macroscopic contact angle, $\theta$, of a liquid droplet on the graphene-coated surface. ${ }^{[23]}$ Effort made in this area can also facilitate development of 2D materials-based membrane and microfluidics that take advantage of the ultra-thinness of 2D material as an ideal molecular barrier.

It is important to recognize that interactions between molecules residing at a $2 \mathrm{D}$ material surface are associated with multiscale physical phenomena, which are extremely complex. Taking the interactions between a water droplet and a graphenecoated surface as an example, as shown in Fig. 3A, continuum physics can describe the macroscopic ( $\sim \mu \mathrm{m}$ scale) contact angle, $\theta$, which is quantitatively determined by the competition between surface and interfacial tensions in the context of the Young-Dupré equation. [24] However, on the nanometer scale, the molecular interactions at the graphene interface are associated with an N-body system, which is more appropriately modeled using MD simulations, resulting in an oscillatory density distribution for liquid molecules adjacent to graphene. ${ }^{[25]}$ If we further zoom in to the graphene interface, quantum physics becomes more dominant. Since there is an energy difference between the lowest unoccupied molecular orbital (LUMO) of water and the $\mathrm{E}_{\mathrm{F}}$ of graphene, a significant degree of electron transfer occurs, such that graphene is ' $p$-doped' by the adjacent water molecules.[26] In this case, water molecules act as 'electron acceptors' to graphene, and depending on the position of the LUMO, different electron donor molecules have also been identified. ${ }^{[26]}$ It is clear that understanding and engineering the multiscale physical phenomena associated with the molecular interactions at 2D materials interface will allow us to manipulate the surface, interfacial, chemical, and electronic properties of 2D materials, which are all essential to fabricate 2D materials-based integrated devices. In my research group, we have been developing mathematical models to bridge different physical phenomena associated with different length scales. An example of modeling wetting behavior of graphene is briefly discussed below.

Graphene has been recognized as the strongest material in the world, based on the obtained maximum force per unit thickness that a material can withstand before breaking. ${ }^{[27]}$ However, due to its ultrathinness, recent findings when characterizing suspended graphene suggest that the surface tension of liquids, ${ }^{[28]}$ as well as the evaporation of liquids, ${ }^{29]}$ can readily break a sheet of freestanding graphene. As a result, in order to determine the wetting behavior of graphene in a precise and reproducible manner, it is necessary to carry out contact angle measurements on a substrate-supported graphene. To this end, one typically needs to transfer the as-prepared graphene from a copper foil, which catalyzes the synthesis of graphene in a CVD furnace, to a target substrate before measuring the contact angle. ${ }^{[30]}$ Consequently, the measured contact angles necessarily include the contributions from both graphene and the underlying substrate, such that the role of graphene in transmitting the wetting behavior of the substrate comes into play. ${ }^{[31]}$

Very recently, by comparing the measured contact angles of water before and after deposition of graphene $(\mathrm{G})$ onto a target substrate (S), namely, $\theta_{\mathrm{S}}$ and $\theta_{\mathrm{GS}}$, respectively, two contradictory conclusions were reached: (1) "monolayer graphene is completely transparent to wetting (that is,

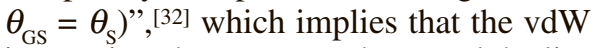
interactions between graphene and the liquid molecules placed on top are negligible, thereby permitting the 'transmission' of the wettability of substrate to fluid sitting on graphene (see Fig. 2A), and (2) "monolayer graphene is completely opaque to wetting, and has a constant $\theta_{\mathrm{GS}} \sim 90^{\circ}$, independent of the underlying substrate" (see Fig. 2B). ${ }^{[33]}$ Interestingly, conclusions (1) and (2) are supported by consistent results of experiments, modeling, and molecular dynamics (MD) simulations. ${ }^{[32,33]}$ Clearly, a more comprehensive model and analysis are necessary to bridge the multiscale physical phenomena, in order to elucidate the wettability of graphene, as well as the role of the underlying supporting substrates.

My recent work in Physical Review Letters is focused on the wettability of water on different graphene species, ${ }^{[24]}$ and shows that both conclusions (1) and (2) do not properly address the wettability of graphene-coated substrate, as well as the interfacial tension at the water/graphene interface. In that work, I developed the first theory to model the short-range vdW potentials between a bulk liquid phase and a film of N-layer graphene (or graphite). Using the experimentally-known contact angle of water on graphite $(\mathrm{N} \rightarrow \infty)$, I developed a model that is able to estimate the water contact angle on a free-standing monolayer $(\mathrm{N}=1)$ graphene. The value is predicted to be $96^{\circ}$, representing the upper limit of water contact angle achievable on any graphene-coated substrate. This theory was further corroborated by carrying out molecular dynamics (MD) simulations considering the contact angle of water on a sheet of free-standing mono-, bi-, and trilayer graphene (Fig. 3B). The expression for the vdW potential between a bulk liquid phase and a film of N-layer graphene supported by a solid surface was also obtained. I demonstrated that monolayer graphene only partially transmitted the wetting property of the underlying substrate 


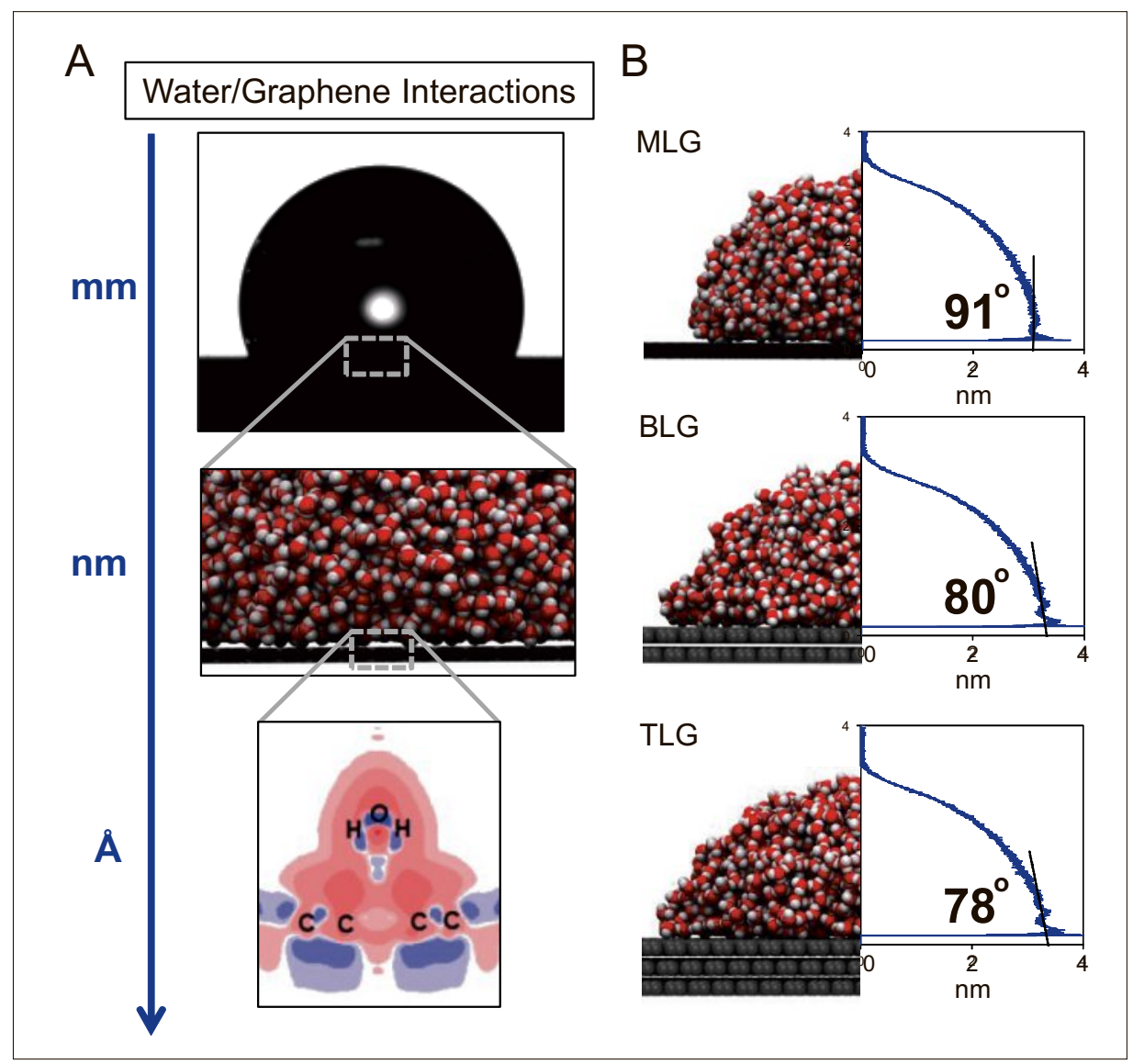

Fig. 3. Wetting phenomena on 2D materials. (A) Multiscale physical phenomena involved at the 2D material-liquid interface. (B) MD simulated water droplets sitting on free-standing mono-, bi-, and trilayer graphene. Reprinted with permission from ref. [31]. Copyright 2013 Nature Publishing Group.

through it, by comparing with the calculated contact angles on bare solid surfaces, and the so-called 'wetting transparency' appears to break down on superhydrophobic and superhydrophilic substrates. In other words, I demonstrate that the degree of wetting 'transparency' or 'opacity' of graphene depends on the substrate choice, with graphene behaving like a 'nonlinearly translucent' film that the liquid droplet sitting on top still interacts with the substrate.

\section{Engineering Electronic Transport at 2D Materials-Semiconductor Interfaces}

With the knowledge developed in stacking-controlled synthesis and interfacial phenomena addressed above, we also devote ourselves to the potential applications of 2D materials-based electronic devices. Integration of these materials into existing semiconductor technologies brings new research opportunities in device design concepts. In this section, we discuss our recent progress in the development of 2D materials-based quantum capacitors (QCs), which are operated based on manipulating electronic transport at the
2D material-semiconductor interface via the field effect.

The field effect refers to the modulation of the space charge concentration in a semiconductor by applying an electric displacement field. Over the past few decades, this effect has been utilized to enable a wide range of metal-oxide-semiconductor (MOS) electronics, including the fieldeffect transistors (FETs) and the floatinggate memory devices. ${ }^{[34]}$ On the other hand, the two-terminal active electronic components, such as diodes, which consist of semiconductor layers sandwiched between two metallic electrodes, fundamentally prohibit introduction of the field effect due to strong electric-field screening in metal. In 1988, Luryi proposed the concept of 'quantum capacitors (QCs)' and discussed the possibility of using a two-dimensional electron gas (2DEG) as one terminal that allows partial penetration of the field effect. ${ }^{[35]}$ The 2DEG was subsequently realized by trapping electrons in a quantum well or confining an inversion layer in a metal-oxide-semiconductor (MOS) capacitor. ${ }^{[36]}$ However, despite great success in demonstration of concept, the 2DEGs are limited in a few materials systems and often require complicated device architecture. In addition to graphene, recent development in other two-dimensional semimetals and semiconductors, such as silicene, germanene, and monolayer transition metal dichalcogenides (TMDs), has further suggested new opportunities in integrating them into different materials systems. Due to the fact that the thickness of these onemolecule-thick materials is comparable to their Debye screening length, they have been considered as the ideal candidates of 2DEG to realize the QC devices on a large scale.

Recently, advanced optoelectronic devices based on the heterostructures that integrate 2D materials and bulk semiconductors have been explored, in which the characteristics can be tuned by the field effect using a gate voltage. ${ }^{[37]}$ For example, device architecture of a vertical transistor of graphene-C60 heterostructure is shown in Fig. 4A. ${ }^{[38]}$ These reports, nevertheless, have attributed the observed gate-tunable behavior to the Fermi level change in the $2 \mathrm{D}$ monolayer sitting on the dielectrics, whereas the field effect penetration through the 2D material is usually oversimplified or ignored. To this end, we presented a multiscale theoretical framework that integrates the $a b$ initio calculated electronic structures and the continuum Poisson-Boltzmann model to inform the field effect penetration in a metal-oxidegraphene-semiconductor (MOGS) quantum capacitor (QC), allowing us to quantify the extent of 'transparency' for the 2D materials as the two-dimensional electron gas (2DEG) to an electrostatic field. It is found that the space charge concentration in the semiconductor layer can be modulated by electrostatic gating, resulting in a thin accumulation or inversion layer at the semiconductor/graphene heterointerface, as shown in Fig. 4B. ${ }^{[39]}$ The extent of transparency to an electrostatic field in a MOGS QC is found to be determined by the combined effect of graphene quantum capacitance and the semiconductor capacitance, thereby allowing prediction of the ranking for a variety of monolayer 2D materials based on their transparency to an electrostatic field as follows: graphene $>$ silicene $>$ germanene $>\mathrm{WS}_{2}>\mathrm{WTe}_{2}>$ $\mathrm{WSe}_{2}>\mathrm{MoS}_{2}>\mathrm{MoSe}_{2}>\mathrm{MoTe}_{2}$, considering electron as the majority carrier. Our findings disclosed a general physical picture of operation modes and design rules for the 2D-materials-based QCs.

In order to justify our theory, we also reported the first experimental evidence that the penetration of field effect through a monolayer 2D material determines the carrier transport at a semiconductor/graphene heterointerface. The new mechanistic insight brought possibilities to rationally design graphene-based vertical field-effect transistors (VFETs). Because both graphene and organic semiconductors 


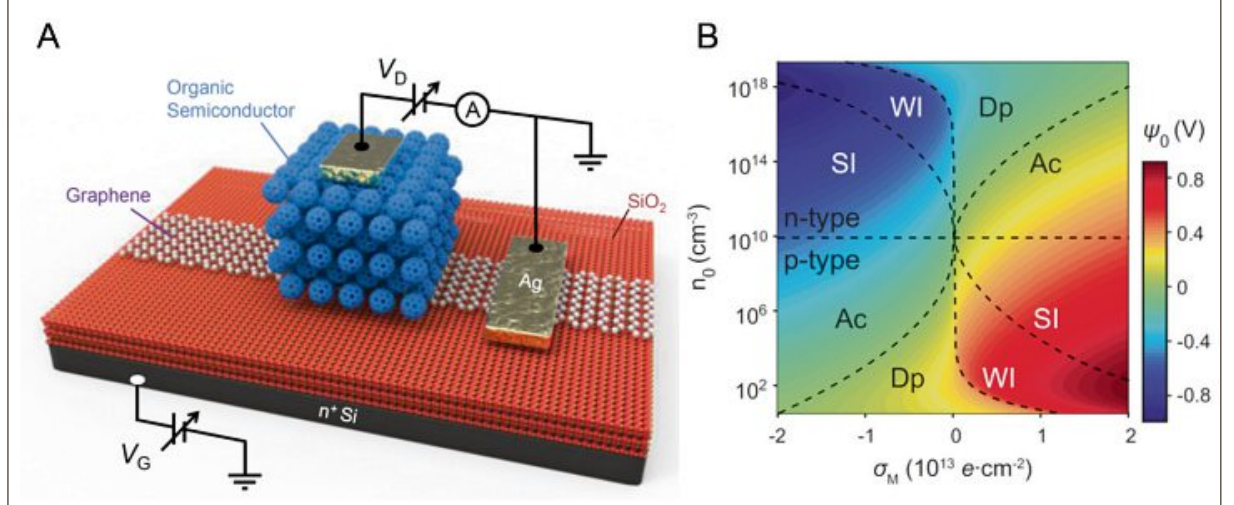

are compatible to the flexible substrate, I further demonstrated flexible organic/ graphene VFETs with a bending radius $<1 \mathrm{~mm}$, with the output current per unit layout area equivalent to that of the stateof-the-art oxide planar FETs.

\section{Conclusions}

In this account, I briefly addressed three representative projects that reflect my research interests. My new group at ETH Zurich will focus on general aspects of surface and interface engineering of nanomaterials. Specifically, the four focused directions are as follows: i) mesoscale modeling of nanomaterials, ii) physics and chemistry at interfaces, iii) nanomaterials processing, and iv) nanomaterials-based devices. By engineering surface chemistry and bridging multiscale physical phenomena at the nanomaterials interfaces, we hope to bring new insights and potential applications to the nanomaterials community.

\section{Acknowledgement}

I am very grateful to my former PhD advisors (Profs. Blankschtein and Strano) at MIT, as well as my postdoc supervisor (Prof. Bao) at Stanford University. I would also like to expresses thanks for financial and technical support from ETH Zurich.

Received: August 29, 2016

[11] P. F. Luckham, S. Rossi, Adv. Coll. Interf. Sci.
[1] S. Z. Butler, S. M. Hollen, L. Y. Cao, Y. Cui, J. A. Gupta, H. R. Gutierrez, T. F. Heinz, S. S. Hong, J. Huang, A. F. Ismach, E. JohnstonHalperin, M. Kuno, V. V. Plashnitsa, R. D. Robinson, R. S. Ruoff, S. Salahuddin, J. Shan, L. Shi, M. G. Spencer, M. Terrones, W. Windl, J. E. Goldberger, ACS Nano 2013, 7, 2898.

[2] V. Nicolosi, M. Chhowalla, M. G. Kanatzidis, M. S. Strano, J. N. Coleman, Science 2013, 340, 1420.

[3] K. S. Novoselov, A. K. Geim, S. V. Morozov, D. Jiang, Y. Zhang, S. V. Dubonos, I. V. Grigorieva, A. A. Firsov, Science 2004, 306, 666.

[4] A. K. Geim, K. S. Novoselov, Nature Mater. 2007, 6, 183.

[5] A. K. Geim, Science 2009, 324, 1530.

[6] K. S. Novoselov, V. I. Fal'ko, L. Colombo, P. R. Gellert, M. G. Schwab, K. Kim, Nature 2012, 490, 192.

[7] D. Golberg, Y. Bando, Y. Huang, T. Terao, M. Mitome, C. Tang, C. Zhi, ACS Nano 2010, 4, 2979.

[8] Q. H. Wang, K. Kalantar-Zadeh, A. Kis, J. N. Coleman, M. S. Strano, Nature Nanotechnol. 2012, 7, 699.

[9] C. C. Coleman, H. Goldwhite, W. Tikkanen, Chem. Mater. 1998, 10, 2794.

[10] R. Z. Ma, T. Sasaki, Adv. Mater. 2010, 22, 5082. $1999,82,43$.

[12] V. Grasso, 'Electronic structure and electronic transitions in layered materials', Springer.com, 1986.

[13] M. Z. Hasan, J. E. Moore, 'Three-Dimensional Topological Insulators', Annual Review of Condensed Matter Physics, Vol. 2, 2011, pp 5578.

[14] K. S. Novoselov, Angew. Chem. Int. Ed. 2011, 50, 6986.

[15] Y. Zhang, T.-T. Tang, C. Girit, Z. Hao, M. C. Martin, A. Zettl, M. F. Crommie, Y. R. Shen, F. Wang, Nature 2009, 459, 820.

[16] C.-J. Shih, A. Vijayaraghavan, R. Krishnan, R. Sharma, J.-H. Han, M.-H. Ham, Z. Jin, S. Lin, G. L. C. Paulus, N. F. Reuel, Q. H. Wang, D. Blankschtein, M. S. Strano, Nat. Nano. 2011, 6 , 439.

[17] J. N. Coleman, Adv. Funct. Mater. 2009, 19, 3680.

[18] S. Park, R. S. Ruoff, Nature Nanotechnol. 2009, 4, 217.

[19] M. S. Dresselhaus, G. Dresselhaus, Adv. Phys. 1981, 30, 139 .
Fig. 4. Engineering electronic transport at a 2D material-semiconductor interface. (A) Device structure of a vertical transistor that is made by a C60-graphene heterostructure. (B) Calculated electrical potential $\psi_{0}$ at a semiconductorgraphene interface as a function of charge applied on the gate electrode $\sigma_{M}$ and the doping level of the semiconductor $n_{0}$, suggesting that graphene allows penetration of the field effect. Reprinted with permission from refs. [38] and [39]. Copyright 2016 American Chemical Society.
[20] D. D. L. Chung, J. Mater. Sci. 2002, 37, 1475.

[21] S. Bae, H. Kim, Y. Lee, X. F. Xu, J. S. Park, Y. Zheng Y, J. Balakrishnan, T. Lei, H. R. Kim, Y. Il Song, Y.-J. Kim, K. S. Kim, B. Özyilmaz, J.-H. Ahn, B. H. Hong, S. Iijima, Nature Nanotechnol. 2010, 5, 574.

[22] R. R. Nair, P. Blake, A. N. Grigorenko, K. S. Novoselov, T. J. Booth, T. Stauber, N. M. R. Peres, A. K. Geim, Science 2008, 320, 1308.

[23] J. N. Israelachvili, 'Intermolecular and surface forces', 3rd edn. Academic Press: Burlington, MA, 2011.

[24] C.-J. Shih, Q. H. Wang, S. Lin, K.-C. Park, Z Jin, M. S. Strano, D. Blamkschtein, Phys. Rev. Lett. 2012, 109, 176101.

[25] C.-J. Shih, S. Lin, M. S. Strano, D. Blankschtein, J. Am. Chem. Soc. 2010, 132, 14638.

[26] C.-J. Shih, G. L. C. Paulus, Q. H. Wang, Z. Jin, D. Blankschtein, M. S. Strano, Langmuir 2012. 28,8579 .

[27] C. Lee, X. D. Wei, J. W. Kysar, J. Hone, Science 2008, 321, 385.

[28] S. C. O'Hern, C. A. Stewart, M. S. H. Boutilier, J. C. Idrobo, S. Bhaviripudi, S. K. Das, J. Kong, T. Laoui, M. Atieh, R. Karnik, ACS Nano 2012, 6, 10130.

[29] K. I. Bolotin, K. J. Sikes, Z. Jiang, M. Klima, G. Fudenberg, J. Hone, P. Kim, H. L. Stormer, Solid State Commun. 2008, 146, 351.

[30] A. Reina, X. T. Jia, J. Ho, D. Nezich, H. B. Son, V. Bulovic, M. S. Dresselhaus, J. Kong, Nano Lett. 2009, 9. 3087.

[31] C.-J. Shih, M. S. Strano, D. Blankschtein, Nat. Mater. 2013, 12, 866.

[32] J. Rafiee, X Mi, H. Gullapalli, A. V. Thomas, F. Yavari, Y. F. Shi, P. Ajayan, N. Koratkar, Nature Mater. 2012, 11, 217.

[33] R. Raj, S. C. Maroo, E. N. Wang, Nano Lett. 2013, 13, 1509

[34] S. M. Sze, K. K. Ng, 'Physics of Semiconductor Devices', John Wiley \& Sons, 2006.

[35] S. Luryi, Appl. Phys. Lett. 1988, 52, 501.

[36] J. H. Davies, 'The Physics of Low-dimensional Semiconductors: An Introduction', Cambridge University Press, 1997.

[37] A. K. Geim, I. V. Grigorieva, Nature 2013, 499, 419.

[38] C.-J. Shih, R. Pfattner, Y.-C. Chiu, N. Liu, T. Lei, D. Kong, Y. Kim, H.-H. Chou, W.-G. Bae, Z. Bao, Nano Lett. 2015, 15, 7587.

[39] T. Tian, P. Rice, E. J. G. Santos, C.-J. Shih, Nano Lett. 2016, 16, 5044. 